\title{
Polynomial energy decay rate and strong stability of Kirchhoff plates with non-compact resolvent
}

\author{
Bopeng $\mathrm{RAO}^{1}$ and Ali WEHBE ${ }^{2}$
}

\begin{abstract}
Using a multiplier method, we establish the polynomial energy decay rate for the smooth solutions of Kirchhoff plates equations. Consequently, we obtain the strong stability in the absence of compactness of resolvent of the infinitesimal operator.
\end{abstract}

Key words. Kirchhoff plates, polynomial decay rate, strong stability, non-compactness.

AMS subject classifications. 93D20, 93B05, 35B35, 35B37.

\section{Introduction and main results}

In this paper we study the stability of a thin elastic plate with dynamical boundary feedback controls. Let $\Omega \subset \mathbb{R}^{2}$ be a bounded open set with smooth boundary $\Gamma$ consisting of a clamped part $\Gamma_{0}$, and a rimmed part $\Gamma_{1}$ such that $\bar{\Gamma}_{0} \cap \bar{\Gamma}_{1}=\{\emptyset\}$. Following the theory of linear elasticity, the vibration of a thin elastic plate is gouverned by the linear system :

$$
\begin{cases}y_{t t}-\gamma \Delta y_{t t}+\Delta^{2} y=0 & \text { in } \Omega \times \mathbb{R}^{+} \\ y=\frac{\partial y}{\partial \nu}=0 & \text { on } \Gamma_{0} \times \mathbb{R}^{+} \\ \Delta y+(1-\mu) B_{1} y+\eta=0 & \text { on } \Gamma_{1} \times \mathbb{R}^{+} \\ \frac{\partial \Delta y}{\partial \nu}+(1-\mu) \frac{\partial B_{2} y}{\partial \tau}-\gamma \frac{\partial y_{t t}}{\partial \nu}+\frac{\partial^{2} y_{t}}{\partial \tau^{2}}-\xi=0 & \text { on } \Gamma_{1} \times \mathbb{R}^{+}\end{cases}
$$

where $\nu=\left(\nu_{1}, \nu_{2}\right)$ denotes the unit normal vector, $\tau=\left(\tau_{1}, \tau_{2}\right)$ the unit tangent vector, and where $\gamma>0$ and $0<\mu<1 / 2$ are physical constants. The boundary

\footnotetext{
${ }^{1}$ Institut de Recherche Mathématique Avancée, Université Louis Pasteur de Strasbourg, 7, rue Réné-Descartes, 67084 Strasbourg Cedex, France. E-mail: rao@irma.u-strasbg.fr

${ }^{2}$ CAMS, College Hall, Room 425, American University of Beirut, P.O.BOX : 110236, Beirut, Lebanon. E-mail: ali_wehbe@yahoo.fr
} 
operators $B_{1}, B_{2}$ are defined by :

$$
\left\{\begin{array}{l}
B_{1} y=2 \nu_{1} \nu_{2} \frac{\partial^{2} y}{\partial x_{1} \partial x_{2}}-\nu_{1}^{2} \frac{\partial^{2} y}{\partial x_{2}^{2}}-\nu_{2}^{2} \frac{\partial^{2} y}{\partial x_{1}^{2}} \\
B_{2} y=\left(\nu_{1}^{2}-\nu_{2}^{2}\right) \frac{\partial^{2} y}{\partial x_{1} \partial x_{2}}+\nu_{1} \nu_{2}\left(\frac{\partial^{2} y}{\partial x_{2}^{2}}-\frac{\partial^{2} y}{\partial x_{1}^{2}}\right) .
\end{array}\right.
$$

In the case of static feedbacks : $\eta=\partial_{\nu} y_{t}, \xi=y_{t}$, the stability of system (1.1)(1.2) was well studied by different approaches (see [7], [9], [10], [11], [18], [23] and the references wherein). In this work, we consider the case of dynamical boundary controls $\eta(t), \xi(t)$ which are given by the following integral system :

$$
\begin{cases}\eta_{t}-\frac{\partial y_{t}}{\partial \nu}+\eta=0 & \text { on } \Gamma_{1} \times \mathbb{R}^{+} \\ \xi_{t}-y_{t}+\xi=0 & \text { on } \Gamma_{1} \times \mathbb{R}^{+}\end{cases}
$$

The concept of dynamical control has been introduced by the automaticians in the finite dimensional case (see Francis [6]). In the infinite dimensional case, the dynamical controls form part of indirect damping mechanisms proposed by Russell [21].

Now let $y$ be a smooth solution of system (1.1)-(1.3). We define the associated energy $E(t)$ by

$$
E(t)=\frac{1}{2}\left\{\int_{\Omega}\left(y_{t}^{2}+\gamma\left|\nabla y_{t}\right|^{2}\right) d x+\int_{\Omega} a(y, y) d x+\int_{\Gamma_{1}}\left(|\eta|^{2}+|\xi|^{2}\right) d \Gamma\right\}
$$

where for smooth functions $y, z$, we have put :

$a(y, z)=\frac{\partial^{2} y}{\partial x_{1}^{2}} \frac{\partial^{2} z}{\partial x_{1}^{2}}+\frac{\partial^{2} y}{\partial x_{2}^{2}} \frac{\partial^{2} z}{\partial x_{2}^{2}}+\mu\left(\frac{\partial^{2} y}{\partial x_{1}^{2}} \frac{\partial^{2} z}{\partial x_{2}^{2}}+\frac{\partial^{2} y}{\partial x_{2}^{2}} \frac{\partial^{2} z}{\partial x_{1}^{2}}\right)+2(1-\mu) \frac{\partial^{2} y}{\partial x_{1} \partial x_{2}} \frac{\partial^{2} z}{\partial x_{1} \partial x_{2}}$.

Then a straightforward computation gives (see [10] and [18]) :

$$
\frac{d E(t)}{d t}=-\int_{\Gamma_{1}}\left(|\eta|^{2}+|\xi|^{2}+\left|\frac{\partial y_{t}}{\partial \tau}\right|^{2}\right) d \Gamma \leq 0
$$

Therefore system (1.1)-(1.3) is dissipative in the sense that the energy is nonincreasing. Moreover, denoting by $u=\left(y, y_{t}, \eta, \xi\right)$ the state of system (1.1)-(1.3), we can formulate the problem as an evolutionary equation :

$$
u_{t}+\mathcal{A} u=0, \quad u(0)=u_{0} \in \mathcal{H}
$$

where $\mathcal{A}$ is a maximal monotone operator in an appropriate Hilbert energy space $\mathcal{H}$. In the one dimensional case [24], we have shown that equation (1.6) has non uniform energy decay rate. So we turn to establish the polynomial energy decay rate for the smooth initial data. There are several approaches about the polynomial energy decay rate. We refer to [12] for wave equation with local intern damping, [15] for abstract systems by semigroup approaches, [19] for hybrids systems and [2] 
for partially damped systems by multipliers, [16] and [14] for Riesz basis approach. In this work, we will adapt a nonlinear method introduced in [19] to the linear equation (1.6) and establish the energy decay rate as $\frac{1}{t}$ for the smooth initial data in $D(\mathcal{A})$. The method is direct and gives the optimal decay rate in the underlying context.

We first establish the following polynomial energy decay rate :

Theorem 1.1 Assume that

$(H 1): \Gamma_{0} \neq \emptyset$ and $\bar{\Gamma}_{0} \cap \bar{\Gamma}_{1}=\emptyset$;

(H2) : There exists $\delta>0$ and $x_{0} \in \mathbb{R}^{2}$ such that, putting $m(x)=x-x_{0}$, we have :

$$
(m \cdot \nu) \leq 0 \quad \forall x \in \Gamma_{0} \quad \text { and }(m \cdot \nu) \geq \delta^{-1}, \quad \forall x \in \Gamma_{1} .
$$

Then for any smooth initial data $u_{0} \in D(\mathcal{A})$, there exist a constant $M>0$ depending only on $\left\|u_{0}\right\|_{D(\mathcal{A})}$ such that the energy $E(t)$ of the system (1.6) has a polynomial decay rate:

$$
E(t) \leq E(0) \frac{2 M}{M+t}, \quad \forall t \geq 0 .
$$

We next consider the strong stability. Notice that the resolvent $(I+\mathcal{A})^{-1}$ is not compact in the energy space $\mathcal{H}$. Therefore, the classic methods such as Lasalle's invariance principle [22] or the spectral decomposition theory of Sz-Nagy-Foias [4] don't work in that case. We have meet some technical difficulties to use more general criteria in [3] and [8] of strong stability of $C^{0}$-semigroup in the absence of compactness of the resolvent. The following result is a consequence of Theorem 1.1 and the contraction of the semigroup $e^{t \mathcal{A}}$.

Theorem 1.2 For any usual initial data $u_{0} \in \mathcal{H}$, the energy $E(t)$ of the system (1.6) asymptotically decreases to zero:

$$
\lim _{t \rightarrow+\infty} E(t)=0 .
$$

The result of Theorem 1.2 is optimal in the sense that the energy $E(t)$ has no uniform decay rate in the general case. Moreover, using a spectral approach, we can prove that the result of Theorem 1.1 is optimal for the one-dimensional problem (see [24]).

\section{Well-posedness of problem.}

Throughout this paper, we assume that $\Gamma=\bar{\Gamma}_{0} \bigcup \bar{\Gamma}_{1}$ of class $C^{4}$ such that $\bar{\Gamma}_{0} \cap \bar{\Gamma}_{1}=\{\emptyset\}$. We first define the following spaces :

$$
\begin{gathered}
X=L^{2}\left(\Gamma_{1}\right), \quad\|\eta\|_{X}^{2}=\int_{\Gamma_{1}}|\eta|^{2} d \Gamma \\
V=\left\{y \in H^{1}(\Omega): y=0 \text { on } \Gamma_{0}\right\}, \quad\|y\|_{V}^{2}=\int_{\Omega}\left(y^{2}+\gamma|\nabla y|^{2}\right) d x,
\end{gathered}
$$




$$
W=\left\{y \in H^{2}(\Omega): y=\frac{\partial y}{\partial \nu}=0 \text { on } \Gamma_{0}\right\},\|y\|_{W}^{2}=\int_{\Omega} a(y, y) d x .
$$

For all $(y, z) \in H^{4}(\Omega) \times H^{2}(\Omega)$, let us recall Green's formula (see [10]):

$$
\begin{gathered}
\int_{\Omega} \Delta^{2} y z d x=\int_{\Omega} a(y, z) d x \\
-\int_{\Gamma}\left(\Delta y+(1-\mu) B_{1} y\right) \frac{\partial z}{\partial \nu} d \Gamma+\int_{\Gamma}\left(\frac{\partial \Delta y}{\partial \nu}+(1-\mu) \frac{\partial B_{2} y}{\partial \tau}\right) z d \Gamma .
\end{gathered}
$$

Now let $y$ be a smooth solution of the system (1.1)-(1.2). Multiplying (1.1) by a function $\phi \in W$ and using Green's formula (2.1), we get :

$$
\int_{\Omega}\left(y_{t t} \phi+\gamma \nabla y_{t t} \nabla \phi\right) d x+\int_{\Omega} a(y, \phi) d x+\int_{\Gamma_{1}} \eta \frac{\partial \phi}{\partial \nu} d \Gamma+\int_{\Gamma_{1}} \xi \phi d \Gamma+\int_{\Gamma_{1}} \frac{\partial y_{t}}{\partial \tau} \cdot \frac{\partial \phi}{\partial \tau} d \Gamma=0 .
$$

Then we define the linear operators $A, D_{2} \in \mathcal{L}\left(W ; W^{\prime}\right), C \in \mathcal{L}\left(V ; V^{\prime}\right), B \in$ $\mathcal{L}\left(X ; V^{\prime}\right)$ and $D_{1} \in \mathcal{L}\left(X ; W^{\prime}\right)$ by

$$
\begin{array}{lr}
<A y, \phi>_{W^{\prime} \times W}=(y, \phi)_{W}, & \forall y, \phi \in W, \\
<B \xi, \phi>_{V^{\prime} \times V}=(\xi, \phi)_{X}, & \forall \xi \in X, \forall \phi \in V, \\
<C y, \phi>_{V^{\prime} \times V}=(y, \phi)_{V}, & \forall y, \phi \in V, \\
<D_{1} \eta, \phi>_{W^{\prime} \times W}=\left(\eta, \partial_{\nu} \phi\right)_{X}, & \forall \eta \in X, \forall \phi \in W, \\
<D_{2} y, \phi>_{W^{\prime} \times W}=\left(\partial_{\tau} y, \partial_{\tau} \phi\right)_{X}, & \forall y, \phi \in W .
\end{array}
$$

By virtue of Lax-Milgram's theorem (see [5]), we see that $A, C$ is the canonical isomorphism from $W$ onto $W^{\prime}$ respectively, from $V$ onto $V^{\prime}$. Thanks to Sobolev's embeddings (see [1]), we check easily that $B, D_{1}, D_{2}$ are continuous operators for the corresponding topologies.

Assume that $A y+D_{1} \eta+D_{2} z \in V^{\prime}$, then we can formulate the variational equation $(2.2)$ as :

$$
y_{t t}+C^{-1}\left(A y+D_{1} \eta+D_{2} y_{t}+B \xi\right)=0, \text { in } V .
$$

We define the energy space $\mathcal{H}=W \times V \times X \times X$, endowed with the usual inner product :

$$
\begin{gathered}
(u, \widetilde{u})_{\mathcal{H}}=\left\{\int_{\Omega}(z \widetilde{z}+\gamma \nabla z \nabla \widetilde{z}) d x+\int_{\Omega} a(y, \widetilde{y}) d x+\int_{\Gamma_{1}}(\eta \widetilde{\eta}+\xi \widetilde{\xi}) d \Gamma\right\}, \\
\forall u=(y, z, \eta, \xi), \quad \widetilde{u}=(\widetilde{y}, \widetilde{z}, \widetilde{\eta}, \widetilde{\xi}) \in \mathcal{H} .
\end{gathered}
$$

Next we introduce the linear unbounded operator $\mathcal{A}$ as follows :

$$
D(\mathcal{A})=\left\{(y, z, \eta, \xi)^{T} \in \mathcal{H}: z \in W \text { and } A y+D_{1} \eta+D_{2} z \in V^{\prime}\right\}
$$




$$
\mathcal{A} u=\left(\begin{array}{c}
-z \\
C^{-1}\left(A y+D_{1} \eta+D_{2} z+B \xi\right) \\
-\frac{\partial z}{\partial \nu}+\eta \\
-z+\xi
\end{array}\right), \quad \forall u \in D(\mathcal{A}) .
$$

Moreover, denoting by $u=\left(y, y_{t}, \eta, \xi\right)^{T}$ the state of the system (1.1)-(1.2), we can formulate the system as an evolutionary equation :

$$
u_{t}+\mathcal{A} u=0, \quad u(0)=u_{0} \in \mathcal{H} .
$$

It is easy to prove that $\mathcal{A}$ is a maximal monotone operator in the energy space $\mathcal{H}$. Then $\mathcal{A}$ generates a $C_{0}$ semigroup $S_{\mathcal{A}}(t)$ of contractions on the energy space $\mathcal{H}$ (see $[5])$.

Proposition 2.1 If $u=(y, z, \eta, \xi) \in D\left(\mathcal{A}^{2}\right)$, then we have:

$$
y \in H^{5 / 2}(\Omega) \cap W .
$$

Proof. Given $u_{0}=\left(y_{0}, z_{0}, \eta_{0}, \xi_{0}\right) \in D(\mathcal{A})$, we solve the equation $\mathcal{A} u=u_{0}$. This means that

$$
\left\{\begin{array}{l}
z=-y_{0} \\
A y+D_{1} \eta+D_{2} z+B \xi=C z_{0} \\
\eta-\frac{\partial z}{\partial \nu}=\eta_{0} \\
\xi-z=\xi_{0}
\end{array}\right.
$$

Since $C$ is the canonic isomorphism from $V$ onto $V^{\prime}$ and since $z_{0} \in V$, then eliminating $z, \eta$ and $\xi$ in (2.8), we find that $y$ satisfies :

$$
A y+D_{1}\left(\eta_{0}-\frac{\partial y_{0}}{\partial \nu}\right)+B\left(\xi_{0}-y_{0}\right)=C z_{0}+D_{2} y_{0}, \quad \text { in } W^{\prime} .
$$

Using Green's formula (2.1), we interpret equation (2.9) into the following variational equation :

$$
\begin{gathered}
\int_{\Omega}\left(\Delta^{2} y+\gamma \Delta z_{0}-z_{0}\right) \phi d x+\int_{\Gamma_{1}}\left[\Delta y+(1-\mu) B_{1} y+\left(\eta_{0}-\frac{\partial y_{0}}{\partial \nu}\right)\right] \frac{\partial \phi}{\partial \nu} d \Gamma \\
-\int_{\Gamma_{1}}\left[\frac{\partial \Delta y}{\partial \nu}+(1-\mu) \frac{\partial B_{2} y}{\partial \tau}-\left(\xi_{0}-y_{0}\right)-\frac{\partial^{2} y_{0}}{\partial \tau^{2}}+\gamma \frac{\partial z_{0}}{\partial \nu}\right] \phi d \Gamma=0, \quad \forall \phi \in W .
\end{gathered}
$$

It follows that

$$
\left\{\begin{array}{l}
\Delta^{2} y=z_{0}-\gamma \Delta z_{0} \in L^{2}(\Omega) \\
\Delta y+(1-\mu) B_{1} y=-\left(\eta_{0}-\frac{\partial y_{0}}{\partial \nu}\right) \in L^{2}\left(\Gamma_{1}\right) \\
\frac{\partial \Delta y}{\partial \nu}+(1-\mu) \frac{\partial B_{2} y}{\partial \tau}=\left(\xi_{0}-y_{0}\right)-\gamma \frac{\partial z_{0}}{\partial \nu}+\frac{\partial^{2} y_{0}}{\partial \tau^{2}} \in H^{-1}\left(\Gamma_{1}\right) .
\end{array}\right.
$$

Then, thanks to the elliptic theory (see [13]), we deduce that $y \in H^{5 / 2}(\Omega)$. The proof is thus complete. 
Proposition 2.2 The resolvent $(I+\mathcal{A})^{-1}$ of the operator $-\mathcal{A}$ is non-compact in the energy space $\mathcal{H}$.

Proof Let $u_{n}=\left(y_{n}, z_{n}, \eta_{n}, \xi_{n}\right)$ be a bounded sequence in $\mathcal{H}$ such that

$$
\left\|u_{n}\right\|_{\mathcal{H}} \leq 1, \quad \forall n \geq 0 .
$$

Let :

$$
\widetilde{u}_{n}=(I+\mathcal{A})^{-1} u_{n}, \quad \forall n \geq 0 .
$$

Then $\widetilde{u}_{n}=\left(\widetilde{y}_{n}, \widetilde{z}_{n}, \widetilde{\eta}_{n}, \widetilde{\xi}_{n}\right)$ is determined by the following system :

$$
\left\{\begin{array}{l}
\widetilde{z}_{n}=\widetilde{y}_{n}-y_{n}, \\
2 \widetilde{\eta}_{n}=\eta_{n}+\frac{\partial \widetilde{z}_{n}}{\partial \nu}, \\
2 \widetilde{\xi}_{n}=\xi_{n}+\widetilde{z}_{n},
\end{array}\right.
$$

where $\widetilde{y}_{n}$ is the unique solution of the variational equation :

$$
\begin{array}{r}
\left(\widetilde{y}_{n}, \phi\right)_{W}+\left(\widetilde{y}_{n}, \phi\right)_{V}+\int_{\Gamma_{1}} \nabla \widetilde{y}_{n} \nabla \phi d \Gamma+\int_{\Gamma_{1}} \widetilde{y}_{n} \phi d \Gamma=\left(y_{n}+z_{n}, \phi\right)_{V} \\
+\int_{\Gamma_{1}} \nabla y_{n} \nabla \phi d \Gamma+\int_{\Gamma_{1}} y_{n} \phi d \Gamma-\int_{\Gamma_{1}} \eta_{n} \frac{\partial \phi}{\partial \nu} d \Gamma-\int_{\Gamma_{1}} \xi_{n} \phi d \Gamma, \quad \forall \phi \in W
\end{array}
$$

from which we get easily that

$$
\left\|\widetilde{y}_{n}\right\|_{W} \leq C\left\|u_{n}\right\|_{\mathcal{H}}<+\infty, \quad \forall n \geq 1 .
$$

The using the trace theorems, we can find positive constant $C$ such that for all $n \geq 1$ we have :

$$
\left\|\frac{\partial \widetilde{z}_{n}}{\partial \nu}\right\|_{H^{1 / 2}\left(\Gamma_{1}\right)}+\left\|\widetilde{z}_{n}\right\|_{H^{3 / 2}\left(\Gamma_{1}\right)} \leq C\left\|\widetilde{z}_{n}\right\|_{W} \leq C\left(\left\|\widetilde{y}_{n}\right\|_{W}+\left\|y_{n}\right\|_{W}\right)<+\infty
$$

Since the imbeddings from $H^{1 / 2}\left(\Gamma_{1}\right)$ and $H^{3 / 2}\left(\Gamma_{1}\right)$ into $L^{2}\left(\Gamma_{1}\right)$ are compact, without lass of generality, we can assume that $\frac{\partial \widetilde{z}_{n}}{\partial \nu}$ and $\widetilde{z}_{n}$ converge strongly in $L^{2}\left(\Gamma_{1}\right)$. Now let $\left(\xi_{n}\right)_{n \geq 0}$ be an orthonormal sequence in $L^{2}\left(\Gamma_{1}\right)$. By Bessel's inequality we know that

$$
\xi_{n} \rightarrow 0, \quad \text { in } L^{2}\left(\Gamma_{1}\right)
$$

and non subsequence of $\xi_{n}$ converges strongly in $L^{2}\left(\Gamma_{1}\right)$. Therefore, non subsequence of $\widetilde{\xi}_{n}=\frac{1}{2}\left(\xi_{n}+\widetilde{z}_{n}\right)$ converges strongly in $L^{2}\left(\Gamma_{1}\right)$. This proves that $(I+\mathcal{A})^{-1}$ is non-compact in $\mathcal{H}$. 


\section{Polynomial energy decay rate and strong stabil- ity.}

In this section, we first establish the polynomial energy decay rate for smooth solution of equation (2.6). Next we prove the strong stability for the weak solution. We assume that there exist $\delta>0$ and $x_{0} \in \mathbb{R}^{2}$ such that, putting $m(x)=x-x_{0}$, we have :

$$
(m \cdot \nu) \geq \delta^{-1}, \quad \forall x \in \Gamma_{1} \text { and }(m \cdot \nu) \leq 0, \quad \forall x \in \Gamma_{0} .
$$

Lemma 3.1 Let $v_{1}, v_{2}, \widetilde{v}_{2}, \hat{v}_{2} \in L^{2}\left(\Gamma_{1}\right)$ and $y$ satisfy the following conditions :

$$
\begin{cases}y \in W, \Delta^{2} y \in L^{2}(\Omega) & \\ \Delta y+(1-\mu) B_{1} y=v_{1} & \text { on } \Gamma_{1} \\ \frac{\partial \Delta y}{\partial \nu}+(1-\mu) \frac{\partial B_{2} y}{\partial \tau}=v_{2}+\frac{\partial \hat{v}_{2}}{\partial \tau}+\widetilde{v}_{2} & \text { on } \Gamma_{1} \\ y=\frac{\partial y}{\partial \nu}=0 & \text { on } \Gamma_{0}\end{cases}
$$

Then we have :

$$
\begin{gathered}
-\int_{\Omega} \Delta^{2} y(m \cdot \nabla y) d x \leq-\frac{1}{2} \int_{\Omega} a(y, y) d x \\
+C_{0} \int_{\Gamma_{1}}\left(\left|v_{1}\right|^{2}+\left|v_{2}\right|^{2}+\left|\hat{v}_{2}\right|^{2}\right) d \Gamma-\int_{\Gamma_{1}} \widetilde{v}_{2}(m \cdot \nabla y) d \Gamma,
\end{gathered}
$$

where $C_{0}$ is a positive constant depending only on the domain $\Omega$.

Proof. Assume that $v_{1}, \hat{v}_{2} \in H^{3 / 2}\left(\Gamma_{1}\right)$ and $v_{2}, \widetilde{v}_{2} \in H^{1 / 2}\left(\Gamma_{1}\right)$. Then we have $y \in H^{4}(\Omega)$. Using Green's formula (2.1) we have :

$$
\begin{gathered}
\int_{\Omega} \Delta^{2} y(m \cdot \nabla y) d x=\int_{\Omega} a(y, y) d x \\
+\int_{\Gamma}\left(\frac{\partial \Delta y}{\partial \nu}+(1-\mu) \frac{\partial B_{2} y}{\partial \tau}\right)(m \cdot \nabla y) d \Gamma-\int_{\Gamma}\left(\Delta y+(1-\mu) B_{1} y\right) \frac{\partial(m \cdot \nabla y)}{\partial \nu} d \Gamma \\
+\frac{1}{2} \int_{\Gamma}(m \cdot \nu)\left\{\left(\frac{\partial^{2} y}{\partial x_{1}^{2}}\right)^{2}+\left(\frac{\partial^{2} y}{\partial x_{2}^{2}}\right)^{2}+2 \mu \frac{\partial^{2} y}{\partial x_{1}^{2}} \frac{\partial^{2} y}{\partial x_{2}^{2}}+2(1-\mu)\left(\frac{\partial^{2} y}{\partial x_{1} \partial x_{2}}\right)^{2}\right\} \cdot
\end{gathered}
$$

Since $y=\frac{\partial y}{\partial \nu}=0$ on $\Gamma_{0}$, it follows that

$$
\begin{gathered}
\nabla y=0, \quad B_{1} y=0, \quad \frac{\partial(m \cdot \nabla y)}{\partial \nu}=(m \cdot \nu) \Delta y \text { on } \Gamma_{0}, \\
\left(\frac{\partial^{2} y}{\partial x_{1}^{2}}\right)^{2}+\left(\frac{\partial^{2} y}{\partial x_{2}^{2}}\right)^{2}+2 \mu \frac{\partial^{2} y}{\partial x_{1}^{2}} \frac{\partial^{2} y}{\partial x_{2}^{2}}+2(1-\mu)\left(\frac{\partial^{2} y}{\partial x_{1} \partial x_{2}}\right)^{2}=(\Delta y)^{2} \text { on } \Gamma_{0} .
\end{gathered}
$$

On the other hand, we have :

$$
\left(\frac{\partial^{2} y}{\partial x_{1}^{2}}\right)^{2}+\left(\frac{\partial^{2} y}{\partial x_{2}^{2}}\right)^{2}+2 \mu \frac{\partial^{2} y}{\partial x_{1}^{2}} \frac{\partial^{2} y}{\partial x_{2}^{2}}+2(1-\mu)\left(\frac{\partial^{2} y}{\partial x_{1} \partial x_{2}}\right)^{2}
$$




$$
\geq(1-\mu)\left\{\left(\frac{\partial^{2} y}{\partial x_{1}^{2}}\right)^{2}+\left(\frac{\partial^{2} y}{\partial x_{2}^{2}}\right)^{2}+2\left(\frac{\partial^{2} y}{\partial x_{1} \partial x_{2}}\right)^{2}\right\} .
$$

Inserting (3.5)-(3.7) into (3.4) gives

$$
\begin{gathered}
\int_{\Omega} \Delta^{2} y(m \cdot \nabla y) d x \geq \int_{\Omega} a(y, y) d x \\
+\int_{\Gamma_{1}}\left(v_{2}+\widetilde{v}_{2}\right)(m \cdot \nabla y) d \Gamma-\int_{\Gamma_{1}} v_{1} \frac{\partial(m \cdot \nabla y)}{\partial \nu} d \Gamma-\int_{\Gamma_{1}} \hat{v}_{2} \frac{\partial(m \cdot \nabla y)}{\partial \tau} d \Gamma \\
+\frac{(1-\mu)}{2 \delta} \int_{\Gamma_{1}}\left\{\left(\frac{\partial^{2} y}{\partial x_{1}^{2}}\right)^{2}+\left(\frac{\partial^{2} y}{\partial x_{2}^{2}}\right)^{2}+2\left(\frac{\partial^{2} y}{\partial x_{1} \partial x_{2}}\right)^{2}\right\} .
\end{gathered}
$$

A direct computation gives

$$
\begin{aligned}
& \left|\frac{\partial(m \cdot \nabla y)}{\partial \nu}\right| \leq\left|\frac{\partial y}{\partial \nu}\right|+R\left\{\left(\frac{\partial^{2} y}{\partial x_{1}^{2}}\right)^{2}+\left(\frac{\partial^{2} y}{\partial x_{2}^{2}}\right)^{2}+2\left(\frac{\partial^{2} y}{\partial x_{1} \partial x_{2}}\right)^{2}\right\}^{\frac{1}{2}}, \\
& \left|\frac{\partial(m \cdot \nabla y)}{\partial \tau}\right| \leq\left|\frac{\partial y}{\partial \tau}\right|+R\left\{\left(\frac{\partial^{2} y}{\partial x_{1}^{2}}\right)^{2}+\left(\frac{\partial^{2} y}{\partial x_{2}^{2}}\right)^{2}+2\left(\frac{\partial^{2} y}{\partial x_{1} \partial x_{2}}\right)^{2}\right\}^{\frac{1}{2}}
\end{aligned}
$$

where $R=\|m\|_{L^{\infty}(\Omega)}$ is the diameter of $\Omega$. Then for any $\lambda>0$, it follows that

$$
\begin{gathered}
\int_{\Gamma_{1}} v_{1} \frac{\partial(m \cdot \nabla y)}{\partial \nu} d \Gamma \geq-\frac{\lambda}{2} \int_{\Gamma_{1}}\left|v_{1}\right|^{2} d \Gamma-\frac{1}{\lambda} \int_{\Gamma_{1}}\left|\frac{\partial y}{\partial \nu}\right|^{2} d \Gamma \\
-\frac{R^{2}}{\lambda} \int_{\Gamma_{1}}\left\{\left(\frac{\partial^{2} y}{\partial x_{1}^{2}}\right)^{2}+\left(\frac{\partial^{2} y}{\partial x_{2}^{2}}\right)^{2}+2\left(\frac{\partial^{2} y}{\partial x_{1} \partial x_{2}}\right)^{2}\right\} d \Gamma \\
\int_{\Gamma_{1}} \hat{v}_{2} \frac{\partial(m \cdot \nabla y)}{\partial \tau} d \Gamma \geq-\frac{\lambda}{2} \int_{\Gamma_{1}}\left|\hat{v}_{2}\right|^{2} d \Gamma-\frac{1}{\lambda} \int_{\Gamma_{1}}\left|\frac{\partial y}{\partial \tau}\right|^{2} d \Gamma \\
-\frac{R^{2}}{\lambda} \int_{\Gamma_{1}}\left\{\left(\frac{\partial^{2} y}{\partial x_{1}^{2}}\right)^{2}+\left(\frac{\partial^{2} y}{\partial x_{2}^{2}}\right)^{2}+2\left(\frac{\partial^{2} y}{\partial x_{1} \partial x_{2}}\right)^{2}\right\} d \Gamma \\
\int_{\Gamma_{1}} v_{2}(m \cdot \nabla y) d \Gamma \geq-\frac{\lambda}{2} \int_{\Gamma_{1}}\left|v_{2}\right|^{2} d \Gamma-\frac{R^{2}}{2 \lambda} \int_{\Gamma_{1}}|\nabla y|^{2} d \Gamma .
\end{gathered}
$$

Inserting (3.11)-(3.13) into (3.8), we obtain that

$$
\begin{gathered}
\int_{\Omega} \Delta^{2} y(m \cdot \nabla y) d x \\
\geq \int_{\Omega} a(y, y) d x+\int_{\Gamma_{1}} \widetilde{v}_{2}(m \cdot \nabla y) d \Gamma-\frac{\lambda}{2} \int_{\Gamma_{1}}\left(\left|v_{1}\right|^{2}+\left|\hat{v}_{2}\right|^{2}+\left|v_{2}\right|^{2}\right) d \Gamma \\
+\left(\frac{(1-\mu)}{2 \delta}-\frac{2 R^{2}}{\lambda}\right) \int_{\Gamma_{1}}\left\{\left(\frac{\partial^{2} y}{\partial x_{1}^{2}}\right)^{2}+\left(\frac{\partial^{2} y}{\partial x_{2}^{2}}\right)^{2}+2\left(\frac{\partial^{2} y}{\partial x_{1} \partial x_{2}}\right)^{2}\right\} d \Gamma-\frac{2+R^{2}}{2 \lambda} \int_{\Gamma_{1}}|\nabla y|^{2} d \Gamma .
\end{gathered}
$$


We obtain (3.3) by taking $\lambda>0$ sufficiently large in (3.14) :

$$
\lambda \geq \frac{4 \delta R^{2}}{(1-\mu)} \quad \text { and } \quad \frac{2+R^{2}}{2 \lambda} \int_{\Gamma_{1}}|\nabla y|^{2} d \Gamma \leq \frac{1}{2} \int_{\Omega} a(y, y) d x, \quad \forall y \in W .
$$

The case $v_{1}, v_{2}, \widetilde{v}_{2}, \hat{v}_{2} \in L^{2}\left(\Gamma_{1}\right)$ can be completed by the standard density arguments (see Lemma 3.1 in Rao [18]). The proof is thus complete.

Lemma 3.2 Let $u_{0} \in D\left(\mathcal{A}^{2}\right)$ and $y$ be a smooth solution of the system (1.1)-(1.3). Then for all $0 \leq S \leq T<\infty$ we have :

$$
\int_{S}^{T} \int_{\Omega} y_{t}^{2} E(t) d x d t+\frac{1}{2} \int_{S}^{T} \int_{\Omega} a(y, y) E(t) d x d t \leq M_{1} E(S) E(0)
$$

where $M_{1}$ is a positive constant depending on $\left\|u_{0}\right\|_{D(\mathcal{A})}$.

Proof. Multiplying the equation (1.1) with $m \cdot \nabla y E(t)$ we obtain :

$$
\begin{gathered}
\int_{S}^{T} \int_{\Omega} y_{t t}(m \cdot \nabla y) E(t) d x d t-\gamma \int_{S}^{T} \int_{\Omega} \Delta y_{t t}(m \cdot \nabla y) E(t) d x d t= \\
-\int_{S}^{T} \int_{\Omega} \Delta^{2} y(m \cdot \nabla y) E(t) d x d t
\end{gathered}
$$

Integrating by parts on the first term in (3.16) gives :

$$
\begin{gathered}
\int_{S}^{T} \int_{\Omega} y_{t t}(m \cdot \nabla y) E(t) d x d t=\left[\int_{\Omega} y_{t}(m \cdot \nabla y) E(t)\right]_{S}^{T}-\int_{S}^{T} \int_{\Omega} y_{t}(m \cdot \nabla y) E_{t}(t) d x d t \\
+\int_{S}^{T} \int_{\Omega} y_{t}^{2} E(t) d x d t-\frac{1}{2} \int_{S}^{T} \int_{\Gamma_{1}}(m \cdot \nu) y_{t}^{2} E(t) d \Gamma d t
\end{gathered}
$$

Using Poincarré's inequality, there exists a constant $C_{1}>0$ such that :

$$
\int_{\Omega} y_{t}(m \cdot \nabla y) d x \leq C_{1} E(t), \quad \forall t \geq 0
$$

Then it follows that :

$$
\begin{array}{r}
{\left[\int_{\Omega} y_{t}(m \cdot \nabla y) E(t) d x\right]_{S}^{T}-\int_{S}^{T} \int_{\Omega} y_{t}(m \cdot \nabla y) E_{t}(t) d x d t} \\
\geq-C_{1}\left(E^{2}(S)+E^{2}(T)\right)+C_{1} \int_{S}^{T} E_{t}(t) E(t) d t \geq-3 C_{1} E^{2}(S) .
\end{array}
$$

Inserting (3.18) into (3.17) gives :

$$
\int_{S}^{T} \int_{\Omega} y_{t t}(m \cdot \nabla y) E(t) d x d t
$$




$$
\geq \int_{S}^{T} \int_{\Omega} y_{t}^{2} E(t) d x d t-3 C_{1} E^{2}(S)-\frac{R}{2} \int_{S}^{T} \int_{\Gamma_{1}} y_{t}^{2} E(t) d \Gamma d t .
$$

Since $u_{0} \in D\left(\mathcal{A}^{2}\right)$, from Proposition 2.1 and semigroupe theory (see [17]), we have :

$$
\left\{\begin{array}{l}
y \in C^{0}\left(\mathbb{R}^{+} ; H^{5 / 2}\right) \cap C^{2}\left(\mathbb{R}^{+} ; W\right) \cap C^{3}\left(\mathbb{R}^{+} ; V\right) \\
\eta, \xi \in C^{2}\left(\mathbb{R}^{+} ; L^{2}\left(\Gamma_{1}\right)\right)
\end{array}\right.
$$

Then we can integrate by parts on the second term in (3.16) :

$$
\begin{gathered}
-\gamma \int_{S}^{T} \int_{\Omega} \Delta y_{t t}(m \cdot \nabla y) E(t) d x d t= \\
-\gamma \int_{S}^{T} \int_{\Gamma_{1}} \frac{\partial y_{t t}}{\partial \nu}(m \cdot \nabla y) E(t) d \Gamma d t+\gamma\left[\int_{\Omega} \nabla y_{t} \nabla(m \cdot \nabla y) E(t)\right]_{S}^{T} \\
-\gamma \int_{S}^{T} \int_{\Omega} \nabla y_{t} \nabla(m \cdot \nabla y) E_{t}(t) d x d t-\frac{\gamma}{2} \int_{S}^{T} \int_{\Gamma_{1}}(m \cdot \nu)\left|\nabla y_{t}\right|^{2} E(t) d \Gamma d t .
\end{gathered}
$$

Using Cauchy-Schwarz's inequality, we get :

$$
\left|\gamma \int_{\Omega} \nabla y_{t} \nabla(m \cdot \nabla y) d x\right| \leq C_{2} E(t), \quad \forall t \geq 0
$$

It follows that

$$
\begin{gathered}
\gamma\left[\int_{\Omega} \nabla y_{t} \nabla(m \cdot \nabla y) E(t) d x\right]_{S}^{T} \geq-2 C_{2} E^{2}(S), \\
-\gamma \int_{S}^{T} \int_{\Omega} \nabla y_{t} \nabla(m \cdot \nabla y) E_{t}(t) d x d t \geq-\frac{C_{2}}{2} E^{2}(S) .
\end{gathered}
$$

Inserting (3.22) and (3.23) onto (3.21) we obtain that

$$
\begin{gathered}
-\gamma \int_{S}^{T} \int_{\Omega} \Delta y_{t t}(m \cdot \nabla y) E(t) d x d t \geq-\gamma \int_{S}^{T} \int_{\Gamma_{1}} \frac{\partial y_{t t}}{\partial \nu}(m \cdot \nabla y) E(t) d \Gamma d t \\
-\frac{5}{2} C_{2} E^{2}(S)-\frac{\gamma R}{2} \int_{S}^{T} \int_{\Gamma_{1}}\left|\nabla y_{t}\right|^{2} E(t) d \Gamma d t
\end{gathered}
$$

On the other hand, using (3.20) we have :

$$
\begin{cases}\Delta^{2} y \in L^{2}(\Omega) & \\ \Delta y+(1-\mu) B_{1} y=v_{1} & \text { on } \Gamma_{1} \\ \frac{\partial \Delta y}{\partial \nu}+(1-\mu) \frac{\partial B_{2} y}{\partial \tau}=v_{2}-\frac{\partial \hat{v}_{2}}{\partial \tau}+\widetilde{v}_{2} & \text { on } \Gamma_{1} \\ y=\frac{\partial y}{\partial \nu}=0 & \text { on } \Gamma_{0}\end{cases}
$$

with

$v_{1}=-\eta \in L^{2}\left(\Gamma_{1}\right), v_{2}=\xi \in L^{2}\left(\Gamma_{1}\right), \widetilde{v}_{2}=\gamma \frac{\partial y_{t t}}{\partial \nu} \in L^{2}\left(\Gamma_{1}\right)$ and $\hat{v}_{2}=-\frac{\partial y_{t}}{\partial \tau} \in L^{2}\left(\Gamma_{1}\right)$. 
Applying Lemma 3.1 to (3.25) we get :

$$
\begin{gathered}
-\int_{S}^{T} \int_{\Omega} \Delta^{2} y(m \cdot \nabla y) E(t) \leq-\frac{1}{2} \int_{S}^{T} \int_{\Omega} a(y, y) E(t) d x d t \\
+C_{0} \int_{S}^{T} \int_{\Gamma_{1}}\left(\eta^{2}+\xi^{2}+\left|\frac{\partial y_{t}}{\partial \tau}\right|^{2}\right) E(t) d \Gamma d t-\gamma \int_{S}^{T} \int_{\Gamma_{1}} \frac{\partial y_{t t}}{\partial \nu}(m \cdot \nabla y) E(t) d \Gamma d t .
\end{gathered}
$$

Using (1.3) we have :

$$
\left|y_{t}\right|^{2}+\left|\nabla y_{t}\right|^{2} \leq 2\left(\left|\eta_{t}\right|^{2}+\left|\xi_{t}\right|^{2}+|\eta|^{2}+|\xi|^{2}+\left|\frac{\partial y_{t}}{\partial \tau}\right|^{2}\right), \quad \text { on } \Gamma_{1} \text {. }
$$

Inserting (3.19), (3.24), (3.26) and (3.27) into (3.16) gives that

$$
\begin{aligned}
& \int_{S}^{T} \int_{\Omega} y_{t}^{2} E(t) d x d t+\frac{1}{2} \int_{S}^{T} \int_{\Omega} a(y, y) E(t) d x d t \leq 3\left(C_{1}+C_{2}\right) E(S) E(0) \\
& +\left[(\gamma+1) R+C_{0}\right] E(S) \int_{S}^{T} \int_{\Gamma_{1}}\left(\eta^{2}+\xi^{2}+\left|\frac{\partial y_{t}}{\partial \tau}\right|^{2}+\eta_{t}^{2}+\xi_{t}^{2}\right) d \Gamma d t .
\end{aligned}
$$

On the other hand, from (1.5) we have :

$$
\int_{S}^{T} \int_{\Gamma_{1}}\left(\eta^{2}+\xi^{2}+\left|\frac{\partial y_{t}}{\partial \tau}\right|^{2}\right) d \Gamma d t=-\int_{S}^{T} E_{t}(t) d t \leq E(S) .
$$

Since $u_{0} \in D\left(\mathcal{A}^{2}\right)$ then differentiating the system (2.6) with respect to the variable $t$ and using (3.29) we have :

$$
\int_{S}^{T} \int_{\Gamma_{1}}\left(\eta_{t}^{2}+\xi_{t}^{2}\right) d \Gamma d t \leq E_{1}(S)
$$

where the energy of high order $E_{1}(t)$ is defined by

$$
E_{1}=\frac{1}{2}\left\|u_{t}(t)\right\|^{2}=\frac{1}{2}\|\mathcal{A} u(t)\|^{2} .
$$

Finally inserting (3.29) and (3.30) into (3.28) we obtain (3.15), with

$$
M_{1}=3\left(C_{1}+C_{2}\right)+\left[(\gamma+1) R+C_{0}\right]\left(1+\frac{E_{1}(0)}{E(0)}\right) .
$$

The proof is complete.

Lemma 3.3 Let $u_{0} \in D\left(\mathcal{A}^{2}\right)$ and $y$ be a smooth solution of the system (1.1)-(1.3). Then for all $0 \leq S \leq T<\infty$, we have

$$
\begin{aligned}
\int_{S}^{T} \int_{\Omega} y_{t}^{2} E(t) d x d t & +\gamma \int_{S}^{T} \int_{\Omega}\left|\nabla y_{t}\right|^{2} E(t) d x d t-\int_{S}^{T} \int_{\Omega} a(y, y) E(t) d x d t \\
& \leq M_{2} E(S) E(0)+\frac{1}{2} \int_{S}^{T} E^{2}(t) d t
\end{aligned}
$$

where $M_{2}$ is a positive constant independent of $u_{0}$. 
Proof. Multiplying equation (1.1) with $y E(t)$ and using (2.1) we obtain that :

$$
\begin{gathered}
\int_{S}^{T} \int_{\Omega} y_{t}^{2} E(t) d x d t+\gamma \int_{S}^{T} \int_{\Omega}\left|\nabla y_{t}\right|^{2} E(t) d x d t-\int_{S}^{T} \int_{\Omega} a(y, y) E(t) d x d t= \\
{\left[\int_{\Omega} y_{t} y E(t) d x\right]_{S}^{T}-\int_{S}^{T} \int_{\Omega} y_{t} y E_{t}(t) d x d t+\gamma\left[\int_{\Omega} \nabla y_{t} \nabla y E(t) d x\right]_{S}^{T}} \\
-\gamma \int_{S}^{T} \int_{\Omega} \nabla y_{t} \nabla y E_{t}(t) d x d t+\int_{S}^{T} \int_{\Gamma_{1}} \xi y E(t) d \Gamma d t+\int_{S}^{T} \int_{\Gamma_{1}} \eta \frac{\partial y}{\partial \nu} E(t) d \Gamma d t \\
+\int_{S}^{T} \int_{\Gamma_{1}} \frac{\partial y_{t}}{\partial \tau} \frac{\partial y}{\partial \tau} E(t) d \Gamma d t .
\end{gathered}
$$

Using Poincarré's inequality, there exists a constant $C_{3}>0$ such that :

$$
\begin{gathered}
{\left[\int_{\Omega} y_{t} y E(t) d x\right]_{S}^{T}-\int_{S}^{T} \int_{\Omega} y_{t} y E_{t}(t) d x d t \leq C_{3}\left(E^{2}(S)+E^{2}(T)\right)} \\
-C_{3} \int_{S}^{T} E_{t}(t) E(t) d t \leq 3 C_{3} E^{2}(S) .
\end{gathered}
$$

Similarly we have :

$$
\gamma\left[\int_{\Omega} \nabla y_{t} \nabla y E(t) d x\right]_{S}^{T}-\gamma \int_{S}^{T} \int_{\Omega} \nabla y_{t} \nabla y E_{t}(t) d x d t \leq 3 C_{4} E^{2}(S) .
$$

Inserting (3.33) and (3.34) onto (3.32) we obtain that

$$
\begin{gathered}
\int_{S}^{T} \int_{\Omega} y_{t}^{2} E(t) d x d t+\gamma \int_{S}^{T} \int_{\Omega}\left|\nabla y_{t}\right|^{2} E(t) d x d t-\int_{S}^{T} \int_{\Omega} a(y, y) E(t) d x d t \leq 3\left(C_{3}+C_{4}\right) E^{2}(S) \\
+\int_{S}^{T} \int_{\Gamma_{1}} \xi y E(t) d \Gamma d t+\int_{S}^{T} \int_{\Gamma_{1}} \eta \frac{\partial y}{\partial \nu} E(t) d \Gamma d t+\int_{S}^{T} \int_{\Gamma_{1}} \frac{\partial y_{t}}{\partial \tau} \frac{\partial y}{\partial \tau} E(t) d \Gamma d t .
\end{gathered}
$$

On the other hand, thanks to Sobolev's imbedding we obtain that

$$
\int_{\Gamma_{1}} y^{2} d \Gamma \leq C_{5} E(t), \quad \int_{\Gamma_{1}}|\nabla y|^{2} d \Gamma \leq C_{5} E(t), \quad \forall t \geq 0 .
$$

Choosing $\varepsilon>0$ sufficiently small and using (3.36) we have :

$$
\begin{gathered}
\int_{S}^{T} \int_{\Gamma_{1}} \xi y E(t) d \Gamma d t \leq \frac{1}{2 \varepsilon} \int_{S}^{T} \int_{\Gamma_{1}} \xi^{2} E(t) d \Gamma d t+\frac{\varepsilon}{2} \int_{S}^{T} \int_{\Gamma_{1}} y^{2} E(t) d \Gamma d t \\
\leq \frac{1}{2 \varepsilon} E(S) E(0)+\frac{\varepsilon}{2} C_{5} \int_{S}^{T} E^{2}(t) d t .
\end{gathered}
$$


Similarly we have :

$$
\int_{S}^{T} \int_{\Gamma_{1}} \eta \frac{\partial y}{\partial \nu} E(t) d \Gamma d t \leq \frac{1}{2 \varepsilon} E(S) E(0)+\frac{\varepsilon}{2} C_{5} \int_{S}^{T} E^{2}(t) d t
$$

and

$$
\int_{S}^{T} \int_{\Gamma_{1}} \frac{\partial y_{t}}{\partial \tau} \frac{\partial y}{\partial \tau} E(t) d \Gamma d t \leq \frac{1}{2 \varepsilon} E(S) E(0)+\frac{\varepsilon}{2} C_{5} \int_{S}^{T} E^{2}(t) d t .
$$

Finally inserting (3.37), (3.38) and (3.39) into (3.35) we obtain that :

$$
\begin{gathered}
\int_{S}^{T} \int_{\Omega} y_{t}^{2} E(t) d x d t+\gamma \int_{S}^{T} \int_{\Omega}\left|\nabla y_{t}\right|^{2} E(t) d x d t-\int_{S}^{T} \int_{\Omega} a(y, y) E(t) d x d t \\
\leq\left[3\left(C_{3}+C_{4}\right)+\frac{3}{2 \varepsilon}\right] E(S) E(0)+\frac{3 \varepsilon}{2} C_{5} \int_{S}^{T} E^{2}(t) d t .
\end{gathered}
$$

Choosing $\varepsilon=\frac{1}{3 C_{5}}$ in (3.40) we obtain (3.31).

Proof of Theorem 1.1. We first assume that $u_{0} \in D\left(\mathcal{A}^{2}\right)$. Combining (3.15) and (3.31) we obtain that :

$$
\int_{S}^{+\infty} E^{2}(t) d t \leq M E(0) E(S), \quad \forall S \geq 0
$$

where $M$ is given by

$$
M=C\left(1+\frac{\left\|u_{0}\right\|_{D(\mathcal{A})}^{2}}{\left\|u_{0}\right\|_{E}^{2}}\right), \text { and } C>0 \text { is independent of } u_{0} .
$$

Thanks to a classical result of Haraux (see [9]) we obtain that

$$
E(t) \leq E(0) \frac{2 M}{M+t}, \quad \forall t \geq 0 .
$$

Finally by the density arguments we prove the polynomial decay rate (3.35) for all $u_{0} \in D(\mathcal{A})$.

Proof of Theorem 1.2. Let $u_{0} \in \mathcal{H}$, there exists a sequence $\left(u_{0}^{n}\right)_{n \geq 0}$ in $D(\mathcal{A})$ converges to $u_{0}$ in $\mathcal{H}$. Then $\forall \varepsilon>0$ there exists $n_{\varepsilon}>0$ such that

$$
\left\|u_{0}-u_{0}^{n_{\varepsilon}}\right\|_{\mathcal{H}}<\frac{\varepsilon}{2} \text {. }
$$

From Theorem 1.1 there exists $T>0$ such that

$$
\left\|S_{\mathcal{A}}(t) u_{0}^{n_{\varepsilon}}\right\|_{\mathcal{H}}<\frac{\varepsilon}{2}, \quad t>T .
$$

This implies that

$$
\left\|S_{\mathcal{A}}(t) u_{0}\right\|_{\mathcal{H}} \leq\left\|S_{\mathcal{A}}(t) u_{0}^{n_{\varepsilon}}\right\|_{\mathcal{H}}+\left\|u_{0}-u_{0}^{n_{\varepsilon}}\right\|_{\mathcal{H}} \leq \varepsilon, \quad \forall t>T .
$$

It follows that

$$
\left\|S_{\mathcal{A}}(t) u_{0}\right\|_{\mathcal{H}} \rightarrow 0, \quad \forall t \rightarrow+\infty .
$$

The proof is thus complete. 


\section{References}

[1] R.A. Adams, Sobolov Spaces, Academic Press, New York 1975.

[2] F. Alabau, P. Cannarsa and V. Komornik, Indirect internal stabilization of weakly coupled evolution equations, J. Evolution Equations 2 (2002), pp. 127150 .

[3] W. Arendt and C. J. Batty, Tauberian theorems and stability of one-parameter semigroups, Trans. Amer. Math. Soc. 306(1988), pp. 127-150.

[4] C.D. Benchimol, A note on weak stabilizability of contraction semigroups, SIAM J. Control Optim. 16 (1978), 373-379.

[5] H. Brezis, Analyse Fonctionelle, Théorie et Applications, Masson, Paris, 1992.

[6] B.A. Francis, $H_{\infty}-$ control theory, Lecture Notes in Control and Sciences (1986).

[7] M.A. Horn, Uniform stabilisation of the Kirchhoff plate equation with boundary conditions containing moments of inertia, Journal of Mathematical Systems, Estimation and Control, 4(1): 39-65, 1994.

[8] F. L. Huang, Strong asymptotic stability of linear dynamical systems in Banach spaces, J. Diff. Eqs., 104(1985), pp. 307-324.

[9] V. Komornik, Exact controllability and stabilization, Masson, Paris, 1994.

[10] J. Lagnese, Boundary Stabilization of Thin Plates, SIAM Studies in Applied Mathematics 10, SIM, Philadelphia, PA. 1989

[11] I. Lasiecka, Asymtotic behavior of solutions to the plate equations with nonlinear dissipation occurring through the shear forces and bending moments, Appl. Math. Optim. 21, 167-190 (1990).

[12] G.Lebeau, Algebraic and geometric methods in mathematical physics, (Kaciveli, 1993), 73-109, Math. Phys. Stud., 19, Kluwer Acad. Publ., Dordrecht, 1996.

[13] J.L. Lions and E.Magenes, Problèmes aux Limites Non-Homogènes et Applications, Vol. 1, Dunod,Paris, 1968.

[14] W. Littman and L. Markus, Some recent results on control and stabilization of flexible structures, Proc. COMCON Workshop, Montpellier 1987.

[15] Z. Liu and B. Rao Characterization of polynomial decay rate for the solution of linear evolution equations, to appear.

[16] P. Loreti and B. Rao Spectral compensation and optimal energy decay rate for partially damped systems, C. R. Acad. Sci. Paris, 337, Série I (2003), 531-536. 
[17] A. Pazy, Semigroups of linear operators and Applications to partial differential equation, Applied Mathematical Sciences, V. 44, Springer-Verlag, 1983.

[18] B. Rao Stabilization of Kirchhoff plate equation in star-shaped domain by nonlinear boundary feedback. Nonlinear Analysis, T.A.M. 20, 605-626 (1993).

[19] B. Rao Stabilization of elastic plates with Dynamical Boundary Control, SIAM J. Control Optim. Vol. 36, No. 1, pp. 148-163, 1998.

[20] D.L. Russell, Decay rates for weakly damped systems in Hilbert space obtained with control-theoretic methods, J.Differential Equations, 19(1975),344-370.

[21] D.L. Russell, A General framework for the study of indirect damping mechanisms in elastic systems, J. Math. Anal and Appl. (1993).

[22] M. Slemrod, Feedback stabilization of a linear system in Hilbert space with an a priori bounded control, Math. Control Signals Systems, 2 (1989), 265-286.

[23] A. Wehbe, Boundary stabilization of the equation of Kirchhoff plates, Progress in partial differential equations, Vol 2, p. 174-188. Editer by H. Amann. Pitman Research Notes in Math. Serie 383. Longman, Horlow 1998.

[24] A. Wehbe, Quelques résultats sur la stabilisation frontière des équations de poutres et de plaques. Thesis Université Louis Pasteur, Strasbourg, France, 1998. 